\title{
REVIEWS AND DESCRIPTIONS OF TABLES AND BOOKS
}

62[C, D].-H. Schubert, R. Haussner \& J. ErLebach, Vierstellige Tafeln und Gegentafeln für logarithmisches und trigonometrisches Rechnen in zwei Farben zusammengestellt, (Sammlung Göschen, Band 81) Walter de Gruyter \& Co., Berlin, 1960, 156 p., $15 \mathrm{~cm}$. Price DM 3.60.

This book, consisting largely of four-place tables, represents a revision by Joachim Erlebach of previous editions (dating back to 1913) by Robert Haussner of Schubert's tables, which first appeared in 1898.

As the full title states, the tables are printed in two colors-logarithms in red and all other material in black (in two previous editions that the reviewer has examined, the colors were brown and blue, respectively). A total of sixteen tables are given. These include 4D common logarithms of numbers from 1 to $10^{4}$, supplemented by $7 \mathrm{D}$ logarithms of primes through $97 ; 4 \mathrm{D}$ common logarithms of trigonometric functions; addition and subtraction logarithms; natural logarithms of integers to 100 , supplemented by $6 \mathrm{D}$ tables of the first ten multiples of $\ln 10$ and $\log \mathrm{e}$; squares and cubes; natural values of the trigonometric functions; values of frequently used mathematical constants, mainly to $6 \mathrm{D}$; conversion tables (to 6D) from sexagesimal to radian measure; and miscellaneous useful tables, such as mortality tables, interest and annuity tables, geographical and astronomical tables, a table of physical properties of materials, and a periodic table of chemical elements.

The table of antilogarithms appearing in earlier editions has been omitted; however, inverse interpolation in the table of logarithms is so simple, because of the small differences, that this deletion is of negligible practical importance.

On the title page of the table of common logarithms appears a footnote explaining the formation of the logarithm tables of both numbers and trigonometric functions by truncation of the corresponding entries in one of the Bremiker editions of Vega's tables. In cases of doubtful rounding to $4 \mathrm{D}$ of the Bremiker $7 \mathrm{D}$ logarithms, the convention was arbitrarily adopted of making the fourth-place digit odd. The reviewer found a total of ten entries in the table of logarithms of numbers that were subjected to this rounding; of these, four are affected by rounding errors. Such slight inaccuracies could have been avoided by referring to available tenplace tables such as those of J. Peters, which were first published in Germany in 1922. These rounding errors appear also in the Schubert-Haussner tables dated 1945; only one such error, however, appears in an edition dated 1917 that the reviewer has examined.

In summary, despite minor flaws the reviewer considers this book to be an unusually extensive and valuable compilation of four-place tables. Their continued usefulness and popularity can be inferred from the numerous editions and printings of this member of the Sammlung Göschen series.

J. W. W.

63[C, L].-ANDres ZAvrotsky, "Construccion de una escala continua de las operaciones aritmeticas," Revista Ciencia e Ingeniería de la Facultad de Ingeniería de la Universidad de Los Andes, Mérida, Venezuela, December 1960, No. 7, p. 38-53. The author studies the function $S(x, y ; n)=L^{-n}\left(L^{n} x+L^{n} y\right)$, where $L^{n} x$ is the iterated logarithm: 


$$
L^{n} x=\log L^{n-1} x ; L^{1} x=\log x .
$$

The function $S(x, y ; n)$ is of interest as a generalized arithmetic operation, since the values 0 and 1 of the parameter $n$ yield $x+y$ and $x \cdot y$, respectively.

In a previous paper [1] tables for $S(x, y ; n)$ were given for $x, y=0(1) 10 ; n=$ $-1(1) 3$, where $L^{n} x$ was defined in terms of logarithms to the base 2 .

In the current paper non-integer values of the parameter $n$ are introduced by putting $L^{n} x=H(G x-1)$, where the mutually inverse operators $H$ and $G$ are defined by $G L x=G x-1$ and $H(x-1)=L H x$.

In this paper, where $L^{n} x$ is defined in terms of natural logarithms, the function $S(x, y ; n)$ is tabulated for $x, y=0(1) 10 ; n=\frac{1}{2}, \sqrt{2}$, and for $x, y=2(1) 10 ; n=\pi$. All tabular entries are given to $5 \mathrm{D}$.

\section{F. Theilheimer}

Applied Mathematics Laboratory

David Taylor Model Basin

Washington 7, D. C.

1. A. Zavrotsky, "Algunas generalizaciones del concepto de campo," Acad. Ciencias Fis. Mat. y Nat., Caracas, Venezuela, Boletin, No. 28, 1946, 1947, 23 p. [See RMT 494, MTAC, v. 3, 1948-1949, p. 97.]

64[F].-L. McKee, C. Nicol \& J. Selfridge, "Indices and power residues for all odd primes and powers less than 2000," an unpublished mathematical table stored on magnetic tape, January 18, 1961.

The computing center at the University of Oklahoma has recently computed a table of indices and power residues for all odd primes and powers thereof less than 2000. The computations were done on a modified IBM 650 and have been stored on magnetic tape. Anyone desiring any portion of this table should write to: Director, Computing Center, University of Oklahoma, Norman, Oklahoma.

\section{Authors' Summary}

65[G].-Richard Bellman \& Marshall Hall, JR., Editors, Proceedings of Symposia in Applied Mathematics, Vol. X, "Combinatorial Analysis," American Mathematical Society, 1960, vi + 311 p., $26 \mathrm{~cm}$. Price $\$ 7.70$.

This book contains the following papers, presented at a symposium on applied mathematics sponsored by the American Mathematical Society and the Office of Ordnance Research three years ago (April 1958).

Marshall Hall, Jr. Current Studies on Combinatorial Designs

R. H. Bruck

D. R. Hughes

A. A. Albert

Quadratic Extensions of Cyclic Planes

L. J. Paige \&

C. B. Tompkins

On Homomorphisms of Projective Planes

Finite Division Algebras and Finite Planes

The Size of the $10 \times 10$ Orthogonal Latin Square Problem

R. P. Dilworth

Some Combinatorial Problems on Partially Ordered Sets

R. J. Walker

An Enumerative Technique for a Class of Combinatorial Problems 
A. L. Whiteman

A. J. Hoffman

A. W. Tucker

H. W. Kuhn

H. J. Ryser

Marvin Marcus \& Morris Newman

A. M. Gleason

D. H. Lehmer

J. D. Swift

Olga Taussky \& John Todd

R. E. Gomory

Richard Bellman

Murray Gerstenhaber

Robert Kalaba

J. D. Foulkes

E. N. Gilbert

M. M. Flood
The Cyclotomic Numbers of Order Ten

Some Recent Applications of the Theory of Linear Inequalities to Extremal Combinatorial Analysis

A Combinatorial Equivalence of Matrices

Linear Inequalities and the Pauli Principle

Compound and Induced Matrices in Combinatorial Analysis

Permanents of Doubly Stochastic Matrices

A Search Problem in the $n$-cube

Teaching Combinatorial Tricks to a Computer

Isomorph Rejection in Exhaustive Search Techniques

Some Discrete Variable Computations

Solving Linear Programming Problems in Integers

Combinatorial Processes and Dynamic Programming

Solution of Large Scale Transportation Problems

On Some Communication Network Problems

Directed Graphs and Assembly Schedules

A Problem in Binary Encoding

An Alternative Proof of a Theorem of König as an

Algorithm for the Hitchcock Distribution Problem

Presumably the papers dealing with the application of computing equipment to attacks on combinatorial problems will be of most interest to readers of Mathematics of Computation. The editors list these as being the papers by Paige and Tompkins, Walker, Gerstenhaber, Flood, Gleason, Lehmer, Swift, Todd, and Gomory. Some of the papers listed are directly computational, that of Lehmer being an example, and some are more remote, Flood's being not concerned directly with any computing instrument but highly influenced by his work on coding the assignment problem for a computer.

The delay in publication has caused many of the papers to appear dated, but even these give some information not available in print elsewhere. The most spectacular example is the paper by Paige and Tompkins; this paper does not incorporate examples to be sought by methods of the paper, but obtained otherwise by work of several authors, R. C. Bose, S. S. Shirkhande, E. T. Parker, and others (mentioned in a footnote at the beginning of the paper). On the other hand, this paper outlines techniques which were then admittedly not feasibly applicable to the complete solution of the problem considered, but which are almost applicable on present day machines.

Other papers, all of which were prepared by well chosen experts in their fields, seem to have greater durability, and this volume is one in which readers will find a great deal of current and useful material concerning combinatorial problems. On the whole, the volume is well worth perusing both for its computational implications and for its general information concerning combinatorial problems. Marshall Hall's opening paper, for example, presents an excellent survey of the field, which is valuable to almost anyone not completely up to date in combinatorial design. 
The reviewer would like to note two improvements which would have greatly increased the value of the book.

A unified bibliography at the end of the volume would be much handier than the separate bibliographies at the ends of the papers. Separate bibliographies are hard to find in the volume, and they are less useful for general reference to find a partially remembered work than a unified bibliography would be. Bibliographies associated with individual papers are somewhat more convenient to users of reprints, but only the most insistent authors seemed to be able to talk the Society into furnishing reprints from this volume. On the whole, the reviewer believes that an integrated bibliography is almost a necessity in such integrated volumes, and he is continually puzzled when they do not appear.

A more demanding task is the preparation of an instructive narrative summary of the type which has been produced in the Annals of Mathematics Studies devoted to games; [1] contains both a fine bibliography and a carefully written and highly lucid introduction. The editors of the present volume did make several interesting and instructive statements in their introduction, but they were not moved to shed the large amount of light they could have on the subject matter and its relevance.

One interesting statement from the introduction might be worth quoting: "What is very attractive about this field of research is that it combines both the most abstract and most nonquantitative parts of mathematics with the most arithmetic and numerical aspects. It shows very clearly that the discovery of a feasible solution of a particular problem may necessitate enormous theoretical advances. Perhaps the moral of the tale is that the division into pure and applied mathematics is certainly artificial and to the detriment of the enthusiasts on both sides. Furthermore, the way in which apparently simple problems require a complex medley of algebraic, geometric, analytic and numerical considerations shows that the traditional subdivisions of mathematics are themselves too rigidly labelled. There is one subject, mathematics, and one type of problem, a mathematical problem."

On the whole, the authors and the editors (the reviewer excepted) have done an excellent job of presenting the status of this rapidly developing field. The book is worth having if only for isolated papers: Lehmer's, for example, showing how to make a computer behave, or the beguiling paper by Taussky and Todd, which attacks problems that would cause any computer and its masters to shudder. The editors, the American Mathematical Society, and the Office of Ordnance Research are to be congratulated on this volume.

The reviewer, who usually shies away from reviewing his own work, undertook this review because of the difficulty of finding other non-contributing reviewers, and because of the dated and unimportant nature of the paper to which he contributed.

\section{B. Tompkins}

Institute for Defense Analyses

Princeton, New Jersey

1. A. W. Tucker \& R. D. Luce, Editors, Contributions to the Theory of Games, Volume IV, Ann. of Math. No. 40, Princeton University Press, Princeton, N. J., 1959. 
66[G].-Kenneth Hoffman \& Ray Kunze, Linear Algebra, Prentice-Hall, Inc., Englewood Cliffs, N. J., 1961, ix + 332 p., 24 cm. Price $\$ 7.50$.

This book was written to provide a text for the undergraduate course in linear algebra at the Massachusetts Institute of Technology. It covers vector spaces, linear equations and transformations, polynomials, determinants, invariant directsum decompositions, the rational and Jordan canonical forms for matrices, inner product spaces, and bilinear forms. The treatment is imbued with the modern axiomatic and abstract spirit but many concrete illustrative examples and exercises are given so that a serious, persevering student can master it. When he has done so, he is well equipped to study not only applied mathematics and theoretical physics but also mathematical analysis.

We heartily recommend the book either as a text or for private study. The authors are evidently teachers of experience and good judgment who know and like their subject. Their aim is high, and only the best students will master the course, but all the students will be the better for the part of it they learn. In these difficult days for students it is well to have texts so well planned and written as this one.

Applied Mathematics Laboratory

F. D. Murnaghan

David Taylor Model Basin

Washington 7, D. C.

67[G].-G. Temple, Cartesian Tensors, John Wiley \& Sons, Inc., New York, 1961, vii +92 p., $19 \mathrm{~cm}$. Price $\$ 2.75$.

This introduction to vector and tensor algebra is well planned and should prove of value to the better than average student. The amount of material covered in less than 100 pages is surprising; in addition to the usual topics, there are chapters on isotropic tensors, spinors, and orthogonal curvilinear tensors. The influence of Weyl is evident in the treatment of isotropic tensors and of Brauer and Weyl in the treatment of spinors. We heartily recommend the book, which is addressed to first year students "pursuing an Honours course in Mathematics or Physics" in England. We found only two points where our instruction of the subject would differ slightly.

First, in the reduction of a symmetric tensor to diagonal form the author discusses the ratio $f(u)=\frac{S_{\alpha \beta} u_{\alpha} u_{\beta}}{u_{\alpha} u_{\alpha}}$ and states that this must assume its lower bound when $\partial f / \partial u_{\alpha}=0$. This assumes, tacitly, that the lower bound is not assumed on the boundary of the region $-1 \leqq u_{\alpha} \leqq 1$. The author's statement that one must consider separately "the cases in which the matrices of $S-\lambda_{i} u$ are of ranks 3,2 or 1" does not apply to Schur's induction method, which is indifferent to possible equalities of the characteristic numbers of $S$.

Second, in the treatment of spinors I would emphasize more the two-valued nature of the correspondence between rotations, $R$, and unimodular 2-dimensional unitary matrices, $U$. Thus, while to each $U$ there corresponds only one $R$, to each $R$ there correspond the two matrices $\pm U$. For me, the displacement, in modern physics, of vectors from their front rank position, in favor of spinors, is due to the fact that the 2-dimensional unimodular unitary group is not a proper representa- 
tion, but rather an ambivalent one, of the 3-dimensional rotation group. Thus, there exist tensors of the unitary group which are not found amongst the tensors of the rotation group, and these tensors, the so-called spin-tensors of the rotation group, have their physical significance; on the other hand, all tensors of the rotation group are found among the tensors of the unitary group.

\section{F. D. Murnaghan}

68 [K].-E. D. Barraclough \& E. S. Page, "Tables for Wald tests for the mean of a normal distribution," Biometrika, v. 46, 1959, p. 169-177.

Let $x$ be normally distributed with unknown mean $\theta$ and known variance $\sigma^{2}$. The Wald sequential test for $\theta=\theta_{0}$ against the alternative $\theta=\theta_{1}\left(\theta_{1}>\theta_{0}\right)$ consists in taking observation $x_{n+1}$ as long as $a<\left(\theta_{1}-\theta_{0}\right) / \sum_{i=1}^{n} x_{i} / \sigma^{2}+n\left(\theta_{0}^{2}-\theta_{1}^{2}\right) / 2 \sigma^{2}$ $<b$; sampling stopping when this relation first fails, with $\theta=\theta_{0}$ accepted if the left-hand inequality fails and $\theta=\theta_{1}$ accepted if the right-hand inequality fails. Let $Z=-a \sigma /\left(\theta_{1}-\theta_{0}\right), h=(b-a) \sigma /\left(\theta_{1}-\theta_{0}\right), P_{-}=$probability of accepting $\theta=\theta_{0}$ when true, $P_{+}=$probability of accepting $\theta=\theta_{0}$ when $\theta=\theta_{1}, N_{-}=$average sample number when $\theta=\theta_{0}, N_{+}=$average sample number when $\theta=\theta_{1}$, and $\mu=\left(\theta_{1}-\theta_{0}\right) / 2$. Table 1 of the Appendix contains $2 \mathrm{D}$ values of $h$ and $Z$ for $P_{+}=.05$, $.10(.10) .70, P_{-}=.95, .99, .995, .999$, and $\mu=.25(.25) 1.00$. The values of $a$ and $b$ are determined by $h$ and $z$. For Table 2 of the Appendix (the same combination of values for $P_{+}, P_{-}, \mu$ occur as for Table 1$), 2 \mathrm{D}$ values are given for $N_{+}$and $N_{-}$. Charts I-IV of the Appendix contain curves of $P_{+}$and $P_{-}$as functions of $h$ and $Z$ for given $\mu$. These charts are obtained directly from Table 1 and can be used to determine the operating characteristics of the test for given $a, b, \sigma, \theta_{0}$, and $\theta_{1}$. Also, a comparison is made between Wald's approximations (to the operating characteristics and the average sample numbers) and the true values, for ten combinations of values for $h, Z, \mu$ (Text-Table 1). The conclusion reached is that Wald's approximations are not acceptably accurate for many applications when $\mu \geqq .25$.

System Development Corporation

J. E. WALSH

Santa Monica, California

69 [K].-A. T. Bharucha-REID, Elements of the Theory of Markov Processes and their Applications, McGraw-Hill Book Co., Inc., New York, 1960, xi + 468 p., $24 \mathrm{~cm}$. Price $\$ 11.50$.

In recent years the notions of probability have become increasingly important in the building of models of the world around us. This is true, for example, in certain of the physical sciences, social sciences, and in the simulation of military and other operations. Sometimes probabilistic notions appear directly as basic ingredients of the model, sometimes indirectly as the result of applying Monte Carlo methods to the solution of certain types of functional equations.

The mathematical abstraction of an empirical process whose development is governed by probabilistic laws is known as a stochastic process. A special class of these processes are Markov processes in which the development subsequent to a time $t$ depends (probabilistically) only upon the state of the process at $t$ and not 
upon its previous history. Their theory has been developed extensively in the past three decades and they have enjoyed increasingly wide application. Although the subject has been masterly treated from an advanced standpoint in Doob's classical Stochastic Processes, there remains a dearth of textbooks in English which are accessible to the beginning graduate student who has not yet mastered the subtleties of modern analysis, especially measure theory. Notable exceptions, for the case of a finite number of possible states, are Feller's highly regarded Introduction to Probability Theory and its Applications and the recent book on Finite Markov Chains by Kemeny and Snell.

The present work is intended as a graduate-level text and reference in applied probability theory. According to the author's preface, its purpose is twofold: "first, to present a nonmeasure-theoretic introduction to Markov processes, and second, to give a formal treatment of mathematical models based on this theory which have been employed in various fields." Further, he states that the prerequisites are "a knowledge of elementary probability theory (the first nine chapters of Feller, say), mathematical statistics (Mood level), and analysis (Rudin level). Some knowledge of matrices and differential equations is also required."

Part I develops the theory of Markov processes $x(t)$, with particular reference to branching stochastic processes. Chapter 1, which lays the foundations for its successors, is devoted to the case wherein $x$ assumes values in a discrete denumerable space, and the parameter $t$ is likewise discrete. In Chapter 2 the stochastic variable $x$ again ranges over a discrete denumerable space, but the parameter $t$ is continuous. In Chapter 3 both $x$ and $t$ are continuous. Each chapter is introduced by a summary paragraph. Thereafter the subject matter is developed carefully, systematically, and lucidly. Each chapter is followed by a list of problems and by a bibliography which is intended to be both complete and up-to-date. In order to make use of the latter, one would need a command not only of the standard languages of science-English, French, German, and Russian-but also of such less familiar languages as Polish and Hungarian.

In Part II it is shown how the theory can be appled to problems in a variety of fields. These fields, together with a partial listing of the topics covered, are:

Biology-Growth of populations, epidemics, theory of gene frequencies

Physics-Cascade processes, radioactive transformations, particle counters, theory of nuclear reactors

Astronomy and Astrophysics-Fluctuations of brightness in the Milky Way, spatial distribution of galaxies, radiative transfer

Chemistry-Reaction kinetics

Operations Research-Queueing theory and some of its applications.

Extensive bibliographies are also given for each of these topics.

The book closes with three appendices concerned with generating functions, Laplace and Mellin transforms, and Monte Carlo methods in the study of stochastic processes.

The reviewer was very favorably impressed by this book. The quality of the printing is excellent, with pleasing page layout and very few printing errors. The author's didactic skill and wide familiarity with the subject matter have resulted in a well motivated, well organized, clearly written text from which a student who 
has the indicated prerequisites can readily gain an introduction to the theory and applications of Markov processes.

R. P. EDDY

Applied Mathematics Laboratory

David Taylor Model Basin

Washington 7, D. C.

70 [K].-R. E. Beckhofer, Salah Elmaghraby \& Norman Morse, "A singlesample multiple-decision procedure for selecting the multinomial event which has the highest probability," Ann. Math. Statist., v. 30, 1959, p. 102-119.

Consider $N k$-nomial trials whose cell probabilities satisfy $p_{1}=\cdots=$ $p_{k-1}=p_{k} / \theta^{*}$. We select that cell into which the most events fall, breaking a tie at random if it occurs. The authors give a $5 \mathrm{D}$ table of the probability of selecting cell $k$, for $k=2,3,4 ; \theta^{*}=1.02(.02) 1.1(.1) 2(.2) 3,10$; and $N=1(1) 30$. An approximation is developed and compared with these values.

J. L. Hodges, JR.

University of California

Berkeley, California

71 [K].-K. G. Clemans, "Confidence limits in the case of the geometric distribution," Biometrika, v. 46, 1959, p. 260-264.

The author obtains confidence limits for estimating $m$, the expected number of trials before a device fails, given the sample mean $\bar{x}$, and $N$, the number of devices. If $N$ devices each are from an identical geometric distribution, the distribution of sample sums will follow a Pascal distribution. Two log-log charts are provided for two-sided $90 \%$ : and $98 \%$. confidence limits for $m, 1 \leqq \bar{x} \leqq 10,000$, and $N=2,5,10$, $15,20,30,50,100$. The charts are based on the exact distribution. For $\bar{x}>10,000$, formulas and tables may be used to determine the confidence limits. For large $N>100$ a special formula is given. Alternatively for large $N$, since sample means are approximately normal, confidence limits for $m$ may be found as solutions of the quadratic equation obtained from $t=\sqrt{N}(\bar{x}-m) \div m(m+1)$, where $t$ is the usual normal deviate for the $\alpha$ percent point.

Space Technology Laboratories, Inc.

L. A. Aroinn

Los Angeles, California

72 [K].-E. T. FEDERIGHI, "Extended tables of the percentage points of Student's t-distribution," J. Amer. Statist. Assn., v. 54, 1959, p. 683-688.

The author states that in using Student's $t$-distribution in testing component parts a need for extending the table of upper percentage points was revealed. The method of calculation of these percentage points is presented, and a table containing these results is given. Let $y_{t}$ be the elementary density for Student's $t$ with $n$ degrees of freedom, and denote $\int_{t_{0}}^{\infty} y_{t} d t$ by $P$. The values of $t_{0}$ are given to $3 \mathrm{D}$ for $P=$ $.25, .10, .05, .025, .01, .005, .0025, .001,5 \times 10^{-4}, 25 \times 10^{-5}, 1 \times 10^{-4}, 5 \times 10^{-5}$, $25 \times 10^{-6}, 1 \times 10^{-5}, 5 \times 10^{-6}, 25 \times 10^{-7}, 1 \times 10^{-6}, 25 \times 10^{-8}, 1 \times 10^{-7}$, and $n$ $=1(1) 30$ (5) $60(10) 100,200,500,10^{3}, 2 \times 10^{3}, 10^{4}$, and $\infty$. It would have been 
advantageous had the large values of $n$ been arranged conveniently for harmonic interpolation, such as $n=60,120,240,480,960$, etc.

\section{A. Aroian}

73[K].-Irwin GuTtman, "Optimum tolerance regions and power when sampling from some non-normal universes," Ann. Math. Statist., v. 30, 1959, p. 926-938.

This paper is concerned with obtaining $\beta$-expectation tolerance regions which are minimax and most stringent (see [1] and [2]) for the upper tail of the single exponential population and for the central part of the double exponential distribution. The single exponential probability density function ( $p d f$ ) is of the form $\sigma^{-1} \exp [-(x-\mu) / \sigma]$ with $x \geqq \mu$, where one or both of $\mu$ and $\sigma$ are unknown. The double exponential $p d f$ is of the form $(2 \sigma)^{-1} \exp (-|x-\mu| / \sigma)$, where $\mu$ is known and $\sigma$ is unknown. The sample values are $x_{1}<\cdots<x_{n} ; \bar{x}=\sum_{i=1}^{n} x_{i} / n$; $s=\sum_{i=2}^{n}\left(x_{i}-x_{1}\right) /(n-1) ; \mu_{0}$ and $\sigma_{0}$ represent known values of $\mu$ and $\sigma$; $t=\sum_{i=1}^{n}\left|x_{i}-\mu_{0}\right|$. Then the optimum tolerance intervals, which are easily identified with the situations considered, are $\left[a_{\beta}\left(\bar{x}-\mu_{0}\right), \infty\right),\left[x_{1}-b_{\beta} \sigma_{0}, \infty\right)$, $\left[x_{1}-c_{\beta} s, \infty\right)$, and $\left[\mu_{0}-d_{\beta} t, \mu_{0}+d_{\beta} t\right]$. Tables I-IV contain $6 \mathrm{D}$ values of $a_{\beta}, b_{\beta}$, $c_{\beta}, d_{\beta}$, respectively, for $n=1(1) 20,40,60$ and $\beta=.75, .90, .95, .99$. The power of tolerance intervals is expressed in terms of parameter $\alpha_{1}$, where $\alpha_{1}$ is determined as the solution of $(\alpha \sigma)^{-1} \int_{I(\beta)} \exp [-(x-\mu) / \alpha \sigma d x=\gamma=$ measure of desirability, for the single exponential case, and from $(2 \alpha \sigma)^{-1} \int_{I(\beta)} \exp (-|x-\mu| \alpha \sigma) d x=\gamma$ for the double exponential case. Here $I(\beta)$ is the tolerance interval considered and $0<\gamma<1$ (large values indicate greatest desirability). Tables V, VI, and VIII contain $7 \mathrm{D}$ values of the power for intervals $\left[a_{\beta}\left(\bar{x}-\mu_{0}\right), \infty\right),\left[x_{1}-b_{\beta} \sigma_{0}, \infty\right)$, $\left[\mu_{0}-d_{\beta} t, \mu_{0}+d_{\beta} t\right]$, respectively, for $n=1(2) 7,10,15,30,60$, and $\beta=.75, .90$, $.95, .99$; likewise for $x_{1} c_{\beta} s$ and Table VII, except that $n=2(2) 10,15,30,60$.

\section{J. E. WaLsh}

1. D. A. S. Fraser \& Irwin Gutpman, "Tolerance regions," Ann. Math. Statist., v. 27,1956, p. $162-179$.

2. Inwin Gutrman, "On the power of optimum tolerance regions when sampling from normal distributions," Ann. Math Statist., v. 28, 1957, p. 773-778.

74[K].-Milos Jilek \& Otakar Likar, "Coefficients for the determination of onesided tolerance limits of normal distribution," Ann. Inst. Statist. Math. Tokyo v. 11,1959 , p. $45-48$.

It is well known that a random sample of size $N$ from a normal universe with mean $\mu$ and variance $\sigma^{2}$ yields one-sided tolerance limits $\left(-\infty, T_{u}\right)$ and $\left(T_{L},+\infty\right)$ each of which includes at least a fraction $\alpha$ of the universe with probability $P$, where

$$
\begin{aligned}
T_{u} & =\bar{x}+k s, \\
T_{L} & =\bar{x}-k s,
\end{aligned}
$$


and where,

$$
\begin{aligned}
& \bar{x}=\sum_{i=1}^{n} x_{i} / n \\
& s^{2}=\sum_{i=1}^{n}\left(x_{i}-\bar{x}\right)^{2} /(n-1),
\end{aligned}
$$

and

$$
\sqrt{n} k=t\left(n-1, u_{\alpha} \sqrt{n}, \dot{1}-P\right) .
$$

Here $t(f, \delta, \epsilon)[1]$ is the $100 \epsilon$ percentage point of the non-central $t$ distribution with $f$ degrees of freedom, $\delta$ is the measure of non-centrality in the definition of $t$, and $u_{\alpha}$ is the $100(1-\alpha)$ percentage point of the unit normal distribution with zero mean.

By use of the tables (especially Table IV) and iteration of the approximations given by Johnson and Welch in [1] the authors obtain values of the coefficient $\sqrt{n} k$ to $4 \mathrm{~S}$, for $n=5(1) 20(5) 50(10) 100(100) 300$, for $P$ and $\alpha=.90, .95, .99$. A method for determination of these coefficients is given in [1], but the calculations are, of course, quite tedious, so that the present tables render a valuable service for practical applications to one-sided tolerance limits.

Columbia University

S. B. Littauer

New York, N. Y.

1. N. S. Johnson \& B. L. WeLch, "Applications of the non-central $t$-distribution," Biometrika, v. 31, 1939, p. 362-389.

75[K].-J. Pachares, "Tables of the upper $10 \%$ points of the Studentized range," Biometrika, v. 46, 1959, p. 461-466.

Let $q=w / s$, where $w$ is a sample range based on $n$ values, and $s$ is an independent estimate of standard deviation based on $m$ values. Then tables of $q^{\prime}$ have been prepared for $\operatorname{Pr}\left(q \geqq q^{\prime}\right)=\alpha$, where $\alpha=.01, .05, .10, n=2$ (1) 20, and $m=1$ (1) $20,24,30,40,60,120, \infty$. Three significant figures are given throughout. The work of Harter [1] has been used in improving the accuracy throughout, particularly for $\alpha=.01$. For $\alpha=.01$ and .05 , these tables correct errors in [2].

University of Minnesota

I. R. Savage

Minneapolis, Minn.

1. H. L. HARTER, The Probability Integrals of the Range and of the Studentized Range, WADC Technical Report 58-484, vols. I \& II, 1959. Office of Technical Services, U. S. Dept. of Commerce, Washington 25, D.C.

2. J. M. MAY, "Extended and corrected tables of the upper percentage points of the 'Studentized' range," Biometrika, v. 39, 1952, p. 192-193. [RMT 1080, MTAC, v. 7, 1953, p. 94]

76[K].-K. C. S. Pillai \& Pablo Samson, JR., “On Hotelling's generalization of $\mathrm{T}^{2}$," Biometrika, v. 46, 1959, p. 160-168.

Let $S_{1} / n_{1}, S_{2} / n_{2}$ denote independent covariance matrices arising from samples of sizes $n_{1}$ and $n_{2}$ from two $p$-variate normal populations, and $U^{(8)}=\operatorname{trace} S_{2}^{-1} S_{1}$, where $s$ is the number of non-zero roots. Two approximations are compared with the 
exact values for the upper 5 and 1 percentage points of $U^{(2)}$ for several values of $m=\left(n_{1}-s-2\right) / 2$ and $n=\left(n_{2}-s-2\right) / 2$. The approximations for the upper 5 and 1 percentage points of $U^{(3)}$ and $U^{(4)}$ are given to 3 or $4 \mathrm{D}$ for $m=0,5$, $n=15(5) 50,60(20) 100$.

University of Minnesota

I. OLKIN Minneapolis, Minn.

77[K].-J. G. SAw, "Estimation of the normal population parameters given a singly censored sample," Biometrika, v. 46, 1959, p. 150-159.

As estimators of the mean and variance of a normal distribution, given an ordered sample $x_{1}<x_{2} \cdots<x_{n}$ censored above $x_{r}$, the author proposes

$$
\begin{aligned}
& \mu^{*}=\bar{x}_{r-1}+(1-\epsilon) x_{r}, \text { where } \bar{x}_{r-1}=\sum_{i=1}^{r-1} x_{i} /(r-1), \\
& \eta^{*}=\alpha \sum_{i=1}^{r-1}\left(x_{i}-x_{r}\right)^{2}+\beta \sum_{i=1}^{r-1}\left(x_{i}-x_{r}\right)^{2}
\end{aligned}
$$

respectively, where $\epsilon$ is chosen to make $\mu^{*}$ unbiased, and $\alpha$ and $\beta$ are chosen to make $\eta^{*}$ unbiased and of minimum variance. To facilitate use of these estimators, three tables are appended. Table 1 consists of entries of the weight factor $\epsilon$ and $\operatorname{Var}\left(\mu^{*} / \sigma\right)$ to $10 \mathrm{D}$ for $1<r<n \leqq 20$. Table 2 contains coefficients of $(n+1)^{-i}$ in series approximations to $\epsilon$ and to $\operatorname{Var}\left(\mu^{*} / \sigma\right)$. Weight factors $\alpha$ and $\beta$ are not tabulated directly, and consequently routine application of the author's estimates may be hampered. However, in order to permit calculation of these factors, Table 3, containing coefficients of $(n+1)^{-i}$ in series approximations to them, has been included. These entries are given to $6 \mathrm{D}$ for $p_{r}=.50(.05) .80$, where $p_{r}=r /(n+1)$.

University of Georgia

A. C. Cohen, Jr.

Athens, Georgia

78[K].-Minoru Siotani, "The extreme value of the generalized distances of the individual points in the multivariate normal sample," Ann. Inst. Statist. Math. Tokyo, v. 10, 1959, p. 183-208.

Let $x_{\alpha}{ }^{\prime}=\left(x_{1 \alpha}, \cdots, x_{p \alpha}\right), \alpha=1, \cdots, n$, be $n$ independent observations from a $p$-variate normal population with mean vector $m^{\prime}=\left(m_{1}, \cdots, m_{p}\right)$ and covariance matrix $\Lambda$, and let $\bar{x}^{\prime}=\left(\bar{x}_{1}, \cdots, \bar{x}_{p}\right)$. The upper $5,2 \frac{1}{2}$, and 1 percentage points of the extreme deviate $\hat{\chi}_{\max D}^{2}=\max _{i}\left[\left(x_{i}-\bar{x}\right)^{\prime} \Lambda^{-1}\left(x_{i}-\bar{x}\right)\right]$ is given to $2 \mathrm{D}$ for $n=3(1) 10(2) 20(5) 30, p=2,3,4$. When $\Lambda$ is unknown, let $L$ be a $p \times p$ matrix whose elements, $l_{i j}$, are unbiased estimates of $\lambda_{i j}$, and have a Wishart distribution with $\nu$ degrees of freedom. The upper $5,2 \frac{1}{2}$, and 1 percentage points of the Studentized extreme deviate $\hat{T}_{\max D}^{2}=\max ^{i}\left[\left(x_{i}-\bar{x}\right)^{\prime} L^{-1}\left(x_{i}-\bar{x}\right)\right]$ is given to 2D for $n=3(1) 12,14, \nu=20(2) 40(5) 60,100,150,200$.

I. OLKIN 
79[L].-L. Fox, Tables of Weber Parabolic Cylinder Functions and Other Functions for Large Arguments, National Physical Laboratory Mathematical Tables

Volume 4, Her Majesty's Stationery Office, London, 1960, iii +40 p., $28 \mathrm{~cm}$. (Paperback) Price 12s. 6d.

In a previous work [1], to tabulate, for instance, $I_{n}(x)$ for large $x$, it was found convenient to write $I_{n}(x)=(2 \pi x)^{-1 / 2} e^{x} F_{n}(x)$ and to tabulate the auxiliary function $F_{n}(x)$ for $1 / x=z=0(0.001) 0.05$. This device is very economical as compared with tabulation as a function of $x$, and the $F_{n}(x)$ entries are easily interpolated. Using the same idea, the present volume gives tables which supplement well-known tables of certain transcendental functions as described below. An introduction describes the methods of computation. For each table second central or modified central differences are provided. In some cases modified fourth-order central differences are also given.

Table 1. The exponential integral

$$
\begin{aligned}
& E i(x)=\int_{-\infty}^{x} t^{-1} e^{t} d t, \quad-E i(-x)=\int_{x}^{\infty} t^{-1} e^{-t} d t \\
& E i(x)=e^{x} F(z), \quad z=x^{-1} .
\end{aligned}
$$

Thus, positive $z$ relates to $E i(x)$; negative $z$, to $E i(-x)$. The function $F$ is tabulated to $10 \mathrm{D}$ for $\pm z=0(0.001) 0.100$.

Table 2. Sine and cosine integrals

$$
\begin{gathered}
S i(x)=\int_{0}^{x} t^{-1} \sin t d t, \quad C i(x)=\int_{\infty}^{x} t^{-1} \cos t d t \\
S i(x)=\frac{1}{2} \pi-P \cos x-Q \sin x, \quad C i(x)=P \sin x-Q \cos x
\end{gathered}
$$

Values of $P, Q$ are given to $10 \mathrm{D}$ for $z=x^{-1}=0(0.001) 0.100$.

Table 3. Airy integrals

The notation follows Miller [2]. Let $z=\xi^{-1}=\frac{3}{2} x^{-3 / 2}$.

$$
\begin{aligned}
A i(x) & =\frac{1}{2} \pi^{-1 / 2} x^{-1 / 4} e^{-\xi} R, \quad B i(x)=\pi^{-1 / 2} x^{-1 / 4} e^{\xi} S \\
A^{\prime} i(x) & =\frac{1}{2} \pi^{-1 / 2} x^{1 / 4} e^{-\xi} W, \quad B^{\prime} i(x)=\pi^{-1 / 2} x^{1 / 4} e^{\xi} X \\
A i(-x)+j B i(-x) & =\pi^{-1 / 2} x^{-1 / 4} e^{-j \theta}(j P-Q) \\
A^{\prime} i(-x)+j B^{\prime} i(-x) & =\pi^{-1 / 2} x^{1 / 4} e^{-j \theta}(j U-V), \quad j=(-1)^{1 / 2}, \theta=\xi+\frac{1}{4} \pi .
\end{aligned}
$$

The values of $R, S, W, X, P, Q, U, V$ are tabulated to $10 \mathrm{D}$ for $z=0(0.001) 0.050$.

Table 4. The error integral

$$
\int_{x}^{\infty} e^{-\frac{1}{2} t^{2}} d t=x^{-1} e^{-\frac{1}{2} x^{2}} S, \quad \int_{0}^{x} e^{\frac{1}{t^{2}}} d t=x^{-1} e^{\frac{1}{3} x^{2}} T
$$

The values of $S$ and $T$ to $10 \mathrm{D}$ are provided for $x^{-2}=z=0(0.001) 0.010$. Since error functions are often used in the form $\int^{x} e^{ \pm t^{2}} d t$, tables based on the latter representation should also have been prepared.

Table 5. Factorial functions

This is a table of the gamma function, its natural logarithm, and derivatives of 
the latter. Let

$$
\begin{gathered}
\Gamma(1+x)=(2 \pi)^{1 / 2} e^{-x} x^{x+1 / 2} f \\
\ln \{\Gamma(1+x)\}=\frac{1}{2} \ln (2 \pi)+\left(x+\frac{1}{2}\right) \ln x-x+g \\
F^{(k-1)}(x)=\frac{d^{k}}{d x^{k}}\{\ln \Gamma(1+x)\}, \quad F^{(0)}(x)=\ln x+f_{2}, \quad x^{-1}=z
\end{gathered}
$$

For each table $z=0(0.01) 0.10$. Tabular value of $f, x f_{2}, x F^{\prime \prime}$, and $x^{2} F^{\prime \prime}$ are given to $10 \mathrm{D} ; g$, to $12 \mathrm{D} ; x^{3} F^{\prime \prime \prime}, x^{4} F^{(4)}$, to $9 \mathrm{D}$.

Table 6. Weber functions

The notation follows Miller [3]. Let

$$
\begin{gathered}
W(a, x)=(2 k / x)^{1 / 2} f \cos \chi, \quad W(a,-x)=(2 / k x)^{1 / 2} f \sin \chi \\
\frac{d}{d x} W(a, x)=-(k x / 2)^{1 / 2} g \cos \psi, \quad \frac{d}{d x} W(a,-x)=-(x / 2 k)^{1 / 2} g \sin \psi, \\
x=\varphi+\frac{1}{4} x^{2}-a \ln x+\frac{1}{2} \varphi_{2}+\frac{1}{4} \pi, \quad \psi=\omega+\frac{1}{4} x^{2}-a \ln x+\frac{1}{2} \varphi_{2}-\frac{1}{4} \pi \\
z=x^{-1}, \quad k=\left(1+e^{2 \pi a}\right)^{1 / 2}-e^{\pi a}, \quad \varphi_{2}=I m \ln \left\{\Gamma\left(\frac{1}{2}+i a\right)\right\} .
\end{gathered}
$$

Values of $f, \varphi, g, \omega$ to $8 \mathrm{D}$ are tabulated for $a=-10(1) 10, z=0(0.005) 0.100$. Values of $k$ and $\varphi_{2}$ are also provided. Table $6 \mathrm{~A}$ gives $8 \mathrm{D}$ values of $\varphi_{2}$ for $a=0(0.05) 2.50(0.1) 10.0$.

Y.L.L.

1. L. Fox, A Short Table for Bessel Functions of Integer Order and Large Arguments, Royal Society Shorter Mathematical Tables, No. 3, Cambridge, 1954. See also Review 37, MTAC, v. 9, 1955, p. 73-74.

2. J. C. P. MILler, The Airy Integral, giving Tables of Solutions of the Differential Equation $y^{\prime \prime}=x y$, British Association Mathematical Tables, Part-Volume B, Cambridge, 1946. See also Review 413, MTAC, v. 2, 1946-47, p. 302.

3. National Physical Laboratory, Tables of Weber Parabolic Cylinder Functions. Computed by Scientific Computing Service Limited; Mathematical Introduction by J. C. P. Miller, Editor. Her Majesty's Stationery Office, London, 1955. See also Review 101, MTAC, v. 10, 1956, p. 245-246.

80[X].-Germund Dahlquist, "Stability and error bounds in the numerical integration of ordinary differential equations," Kungl. Tekn. Högsk. Handl. Stockholm (Transactions of the Royal Institute of Technology, Stockholm, Sweden) Nr. 130, 1959, 85 p., 25 cm. Price Kr. 9.

In the first part of his thesis, the author investigates stability of certain linear operators,

$$
L=\rho(E)-h^{r}\left(d^{r} / d x^{r}\right) \sigma(E)+h^{r+1}\left(d^{r+1} / d x^{r+1}\right) \tau(E),
$$

associated with numerical integration formulas for sets of differential equations of the form

$$
d^{r} \bar{y} / d x^{r}=\bar{f}(x, \bar{y}),
$$

where $\bar{y}$ and $\bar{f}$ are s-dimensional vectors, $\rho(\zeta), \sigma(\zeta)$, and $\tau(\zeta)$ are polynomials of degree $k$ with real coefficients, and $E$ is the displacement operator defined by $E u(x)=u(x+h)$, for any function $u(x)$. The number $k$ is called the order of $L$. 
There exists an integer, $p$, called the degree of $L$, such that $L u(x)=O\left(h^{p+r}\right)$ as $h \rightarrow 0$. $L$ is said to be stable if all zeros of $\rho(\zeta)$ are of modulus $\leqq 1$, and the zeros with modulus 1 are of multiplicity $\leqq r$.

The author shows that stability of $L$ is equivalent to "stable convergence" of certain solutions of the difference equation associated with $L$ to the solution of equation (2). If $L$ is unstable the numerical solutions are "strongly unstable," and the integration formula is practically useless. Several theorems are given concerning the largest possible degree of stable operators of a given order. For example, if $\tau(\zeta) \equiv 0, L$ is unstable if $p>2([k / 2]+[(r+1) / 2])$; here $[x]$ denotes the largest integer $\leqq x$.

If the initial-value problem, $d y / d x=q y, y(0)=1, q$ constant, is treated numerically with a stable operator $L$ of order $k$ and degree $k+2$, where $\tau(\zeta) \equiv 0$, the solution is a linear combination of basic solutions, $\zeta_{j h}^{n}, 1 \leqq j \leqq k, n=0,1$, $2, \cdots$, where $\zeta_{j h}$ are the roots of the characteristic polynomial, $\rho(\zeta)-q h \sigma(\zeta)$. Let $\zeta_{j}$ be the roots of $\rho(\zeta)$; in the present case, $\left|\zeta_{j}\right|=1$ and $\zeta_{j}$ is single for all $j$. The author shows that, for $h \rightarrow 0, \zeta_{j h} \sim \zeta_{j}(1+k, q h)$, where the $k_{j}=\sigma\left(\zeta_{j}\right) /$ $\left[\zeta_{j} \rho^{\prime}\left(\zeta_{j}\right)\right]$ are called growth parameters and are real numbers, then $\zeta_{j h}^{n} \sim \zeta_{j}^{n} \exp \left(k_{j} q h n\right)$. One of the $\zeta_{j}$, say $\zeta_{1}$, is equal to 1 , and $k_{1}=1$.

If $\operatorname{Re}(q)<0$, the solution $\zeta_{1, h}^{n} \sim e^{q h_{n}}$ decays, but may be dominated by some of the other basic solutions which increase or decrease more slowly. If there exist solutions which increase for $\operatorname{Re}(q)<0$, the operator $L$ is called weakly unstable; this occurs if at least one of the $k_{j}, j \geqq 2$, is $<0$. It is shown that a stable operator of even order $k$ and maximum degree $k+2$ is weakly unstable; such an operator generates an oscillatory solution whose amplitude increases at least as rapidly as $|\exp (-q n h / 3)|$ if $\operatorname{Re}(q)<0$.

In the second part of this thesis the author is concerned with estimating the norm of the error vector for $r=1$. First, the error is evaluated for the linear variational system $d \bar{z} / d x=B(x) \bar{z}$ associated with (2), where $B(x)$ is the Jacobian, $(\partial \hat{f} / \partial \bar{y})_{\bar{v}=\bar{y}(x)}$. Then the effect of the linearization is estimated separately. Three error formulas are given. The second one, involving the directional derivative, $\mu[B(x)]=\lim _{\lambda \rightarrow 0+} \lambda^{-1}[\|I+\lambda B(x)\|-1]$, yields particularly good results if the numerical solution is smooth and if $\mu[B(x)]<0$.

IBM Research Center

WERNER LiNiger

Yorktown Heights, New York

81[X].-A Korganoff, with the collaboration of L. BossetT, J. L. Groboillot \& J. Johnson, Méthodes de Calcul Numérique, Tome I: Algèbre non linéaire, Dunod, Paris, 1961, xxvii + 375 p., $25 \mathrm{~cm}$. Price $58 \mathrm{NF}$.

The volume being reviewed is the first of a projected series. The characteristicvalue problem is included, but matrix inversion is not, except to the extent that an iterative method that applies to a system of nonlinear equations would apply also to a system of linear equations.

The French literature on numerical analysis is sparse indeed, and hitherto has been but slightly affected by the advent of the electronic computer. This book goes far toward filling the gap there, and would be a substantial contribution to the literature in any language. 
A short initial chapter discusses digital computers and programming in rather general terms. Chapter 2, "Erreurs," consisting of 70 pages, is perhaps the most complete and systematic single account in existence, discussing both rigorous bounds and probabilistic estimates. Chapter 3 considers iterative methods in general as applied to transcendental equations and to systems of equations. Chapter 4 is devoted to polynomials specifically, and Chapter 5 to characteristic values. Each chapter is supplemented by an extensive bibliography. An appendix gives numerical result's of applying the various methods to some specific problems.

One can find fault with a few details. The method of Graeffe is given in the chapter on polynomial equations, although it is equally applicable to transcendental equations within a circle of analyticity. In the chapter on the characteristic-value problem, there is an interesting general section which derives the Jordan normal form, and develops the standard localization theorems of Gershgorin, Brauer, and others. But the treatment of the methods is somewhat disappointing, especially of the so-called "direct methods" (actually methods of reduction, although the reviewer himself is guilty of having propagated the misnomer). It is even stated that the method of Krylov is not to be recommended for large matrices, whereas it has been shown by Bauer and this reviewer that most of the known direct methods are only specializations of the Krylov method.

However, the authors could justify the rather sketchy treatment of the methods of reduction by arguing that these do not differ basically from methods of inversion, and are hence peripheral to their main interest, which would be the solution of the polynomial equation that can be obtained from a complete reduction. The treatment of this problem is by no means sketchy. In fact, it is the most complete and up-todate account of known methods now in the literature, exhibiting well their interrelations, and often presenting them from a fresh point of view. This volume can be recommended highly, and one can hope that the subsequent ones will be equally well done.

Oak Ridge National Laboratory

A. S. Householder

Oak Ridge, Tennessee

82[X].-Herbert E. Salzer, Dexter C. Shoultz, \& Elizabeth P. Thompson, Tables of Osculatory Integration Coefficients, Convair (Astronautics) Division of General Dynamics Corporation, San Diego, 1960, 43 p., 28 cm. (Paperback)

This publication contains tables to implement the use of quadrature formulas of the so-called osculating type, i.e., formulas in which the value of the integrand function and of its derivative at each point are used. The explicit formula considered is

$$
\int_{x_{0}+q h}^{x_{0}+p h} f(u) d u=h \sum_{i=-[(n-1) / 2]}^{[n / 2]}\left\{C_{i}^{(n)}(p) f_{i}+D_{i}^{(n)}(p) h f_{i}^{\prime}\right\}+R_{2 n}(p) .
$$

The authors treat $n=2,3,4,5$; for $n=2$ and $4, q$ is taken to be $\frac{1}{2}$, and for $n=3$ and $5, q$ is taken to be 0 . These choices of $q$ permit use of symmetry relations to reduce the amount of tabulation. The coefficients $C_{i}^{(n)}(p)$ and $D_{i}^{(n)}(p)$ then are polynomials in $p$, and these are listed for each $i$ indicated in (1). Ten-decimal tables 
of $C_{i}^{(n)}(p)$ and $D_{i}^{(n)}(p)$ are given for the same values of $i$ and for $p=q(0.01)[n / 2]$, and $n=2(1) 5$. Tables to aid in computation of an error bound are given.

The authors state that these tables were calculated on an IBM 704 and listed to 12 decimal places. These were subjected to "functional" checks using a desk calculator, and then were rounded to 10 decimal places. On the basis of these checks the authors believe the coefficients are all correct within about one unit in the tenth decimal place.

It seems to the reviewer an unfortunate circumstance that the details of the checks made with the IBM 704 and the desk calculator were not more precisely given. It would have been simple, for example, to have checked the integrals of monomials in the range of precision, and this would have made an excellent independent check of accuracy, well worth the additional time required for automatic calculation.

The authors express great enthusiasm over the accuracy of the method and illustrate it with four examples which indicate rather well the situations in which osculating formulas may be used. These situations are ones in which the derivative of the integrand is available without excessive additional work. In particular, the suggested applications to orbit calculations in which the position and velocity vectors are known seemed very appropriate. However, this very example also suggests that there would be some real interest in extending the domain of $p$ so that extrapolations could be made. In that case $n=1$ would have been a possible choice.

Mrs. Frieda Cohn of the Numerical Analysis Laboratory at the University of Wisconsin has calculated the integrals of a few monomials using these tables, and found that these checked within possibilities of round-off error.

P. C. H.

83[X, Z].-F. A. Ficken, The Simplex Method of Linear Programming, Holt, Rinehart $\&$ Winston, Inc., New York, 1961, vi +58 p., 24 cm. Price $\$ 1.50$.

The book literature on the subject of linear programming is in a state of rapid increase and Ficken's contribution on the simplex method, the essence of the success of linear programming, is a welcome addition. It provides a rigorous treatment of old ideas in a 36-page discussion of duality, feasibility, boundedness, consistency, the simplex tableaux, degeneracy, etc. Brief material on inequalities, linear spaces, matrices, etc., appear in one appendix and theorems on existence and duality in a second appendix. The author's bibliography makes no specific mention of G. Dantzig, to whom the topic of the book owes its existence and who has contributed immeasurably to the development of linear programming. A second point is the absence of material on integer programming. It seems important that a book on the subject, appearing in 1961, should shed light on this significant development. The author has avoided mention of this new gem in which the simplex process plays an important role. Otherwise, the book is recommended for the material it treats and for its clarity and rigor.

Thomas L. SaAty

Office of Naval Research

Navy Department

Washington, D. C. 
Editorial Note: The author wishes to state that subsequent to the publication of this book he learned that the simplex method can be attributed to Dr. G. B. Dantzig. Accordingly, he wishes to extend appropriate acknowledgment along with sincere regret for this unfortunate omission.

84[Z].-C. J. Bouwkamp, A. J. W. Duijvestijn \& P. Medema, Tables Relating to Simple Squared Rectangles of Orders Nine through Fifteen, Department of Mathematics and Mechanics, Technische Hogeschool, Eindhoven (Netherlands), August 1960, ii +360 p., $19 \mathrm{~cm}$.

The problem of dividing a rectangle into a finite number of non-overlapping squares is associated with the problem of dividing a square into smaller squares, all different in size. The latter had been a fascinating but unsolved problem until two solutions were published independently. The first was by R. Sprague (Mathematische Zeitschrift, v. 45, 1939, p. 607) and the second by R. L. Brooks, C. A. B. Smith, A. H. Stone and W. T. Tutte (Duke Mathematical Journal, v. 7, 1940, p. 312-340). The latter paper accomplished this dissection into as few as 26 squares. Later papers by T. H. Willcocks (Fairy Chess Review, v. 7, 1948, and Canadian Journal of Mathematics, v. 3, 1951, p. 304-308) exhibited a dissection into 24 squares. It is not known whether a dissection into fewer squares exists or not. Part of the motivation for the present work is the search for such a dissection.

Each of the foregoing dissections contained a subset of squares which formed a rectangle. A dissection of a rectangle which does not include a rectangular subset is said to be simple; and one in, which no square is repeated is said to be perfect.

Many dissections of rectangles have been derived and published in a series of papers. However, a systematic catalog of these dissections had been prepared only as far as 14 squares. C. J. Bouwkamp, who has been a principal investigator in this field, has collaborated with the other two authors to extend the work to 15 squares and to produce this book, which summarizes the known results. There are only two pages of explanatory text and the rest of the book is devoted to the tables. Table I is a list of the dissections of the 3663 simple perfect rectangles which contain up to 15 squares. They are ordered by increasing values of the ratio of the sides of the rectangle. Table III contains the same rectangles, but they are listed by increasing values of the number of squares. Table II lists the 342 pairs of rectangles which have the same ratio of sides. Of these, 13 pairs have no squares in common. Hence, a nonsimple perfect squaring of a square is made by combining a pair of these rectangles with two squares whose edges are the dimensions of the rectangle. Table IV lists all of the 431 simple imperfect squared rectangles. Of these, four are square.

Several peculiar properties of some of the items in these tables are mentioned. The computation of these rectangles was done on the IBM 650. The details of the analysis and the programming will be published later.

One might consider the extension of these tables to 23 squares to settle the question of the smallest number of unequal squares which make a square. The reviewer estimates that this would require 16,000 books like this one!

Michael Goldberg

Bureau of Naval Weapons

Washington, D. C. 
85[Z].-Food \& Agriculture Organization of the United Nations, Handbook on

Data Processing Methods, Columbia University Press, New York, 1959, vi + 111 p., $27 \mathrm{~cm}$. Price $\$ 1.00$ (Part 1, Provisional Edition) Paperback.

This handbook is a well-written elementary discussion of the methods of processing statistical data from surveys or censuses. It reviews the various methods of data processing, with emphasis on hand and punch card methods. Only one page is devoted to stored program computers. A bibliography is included with the discussion of each topic. The general principles concerned with planning, organizing, administering, and operating data-processing services are adequately covered. Detailed instructions are given for the proper handling and preservation of records and for sorting records, using manual or punch card methods. The handbook is intended for statisticians or supervisors of data processing activities, and hence does not cover the special operating features and details of available machines. Although prepared primarily for the use of statisticians in countries unfamiliar with present dataprocessing tools and procedures, it could serve as a useful introduction to the subject for others.

Bureau of Ships

A. Eugene Smith

Navy Department

Washington 25, D. C.

86[Z].-Fritz Rudolf Güntsch, Einführung in die Programmierung Digitaler Rechenautomaten, Walter de Gruyter \& Co., Berlin, 1960, 144 p., 24 cm. Price DM 24.

This introduction to the programming of digital computers assumes very little previous knowledge of computational procedures. The various steps in the preparation of a computer program are explained with one particular computer, the Z22, in mind. A large number of specific problems are worked out in considerable detail by giving the algorithm, the flow chart, and the detailed coding. Much attention is paid to the discussion of loops, and subroutines are treated with considerable detail. Since the machine code of the Z22 contains only very few instructions-not even a multiplication instruction-nearly all practical coding has to be done in an external code. Therefore, all the illustrative coding in the book up to the second-to-the-last chapter is done in an external code.

The book is devoted exclusively to introducing the reader to programming. It, therefore, does not contain any chapters on numerical analysis or the details of machine logic.

\section{F. Theilheimer}

87[Z].-Allen Kent, Editor, Information Retrieval and Machine Translation, Part 1, Interscience Publishers, Inc., New York, 1960, xv +686p., $23 \mathrm{~cm}$. Price $\$ 23.00$.

This book contains twenty-one of the papers presented at the International Conference for Standards on a Common Language for Machine Searching and Translation, sponsored by Western Reserve University and the Rand Development Corporation, and held in Cleveland, Ohio, September 6-12, 1959. It is the first part of Volume III (in two parts) of the series entitled Advances in Documenta- 
tion and Library Science. The papers, plus discussions and numerous references, afford a good indication of work now in progress on mechanizing the storage and retrieval of information, particularly as it relates to scientific documentation.

The first article (by Allen Kent) reviews the current state of the art in machine literature-searching and translation. It includes in tabular form a comprehensive survey of searching devices and analytical techniques under investigation. Additional tables provide a significant sampling of the recent literature bearing on machine translation. These are arranged according to institution and investigator, subject field, languages involved, and type of equipment used. It is interesting to note that either English or Russian is the target language in nearly every one of the more than one hundred different investigations cited, while the most frequent source languages are Russian, English, French, German, Chinese, and Japanese, in approximately that order of frequency.

The remainder of the book is devoted to topics connected with information retrieval. The papers are by workers from such fields as library science, patent law, engineering, chemistry, medicine, the behavioral sciences, linguistics, symbolic logic, and computer technology. The majority of them deal with problems in organizing, indexing, and coding information for mechanical processing.

The proclivities of each discipline are revealed by the varied approaches to the problems. The librarian is interested in enumeration of characteristics which serve to describe and classify things; the patent searcher is concerned primarily with structure and function, and he needs elaborate means for specifying interrelationships among assemblages of components; the chemist must be able to search in terms of properties, composition, and arrangement; the medical researcher struggles to elucidate information through techniques of statistical analysis and correlation of recorded observations; the linguist has to do with syntactic and semantic analysis of messages to discover the intentional as well as intensional implications which they convey; the logician manipulates information in symbolic form and tries to formulate a consistent set of rules for drawing valid inferences and selecting optimum strategies in a given frame of reference; and the systems designer seeks to provide a means for satisfying the multitudinous requirements of the potential users on an economic basis. Such a diversity of emphasis is characteristic of a field which is evolving rapidly.

Nevertheless, the problems of indexing and searching technical literature, patents, chemical structural formulas, medical records, etc., do have a common basis, even though they may differ in a number of nonessential features. There is general agreement that some kind of coordination of descriptors is necessary for efficient retrieval, but that something more sophisticated than simple conjunction of characteristics will be required to cope with the increasing size and complexity of subject matter files. More particularly, it has been found in encoding subject headings that the relations expressed by the product, sum, and difference of classical logic are not sufficient by themselves for achieving the desired degree of specificity. A reasonably complete representation also requires a means for denoting the syntactic relations between descriptors.

A number of schemes are discussed, particular importance being attached to the rules for analyzing subject matter and to the notation for expressing the results of the analysis. These include the use of modulants, interfixes, and role indicators to 
specify the types of relationships between terms and the use of phrase boundaries, parentheses, or other special marks of punctuation to segregate groups of associated terms. Such devices greatly increase the discriminating power of an index by preventing false associations of terms.

However, there is an evident lack of agreement on the means for attaining these desirable ends. This stems from difficulties inherent in linguistic analysis. It is necessary to discover and to define the syntactic relationships which are significant in mechanical indexing and searching. These are sometimes elusive, although such categories as species, genus, part, whole, factor, process, product, and attribute are widely recognized. The problem of synonyms (or near synonyms) and recognition of specific-generic connections can best be handled through the preparation of technical dictionaries and thesauri. These will provide a semantic analysis of complex notions in terms of more elementary concepts.

Some concern is voiced that the potential gain in accuracy of representation may be offset by an increase in ambiguity of indexing as a result of the greater complexity of the task. No procedure can be considered satisfactory unless there is a reasonable prospect that two different workers indexing an item will arrive at substantially the same description. Further research on the development of information retrieval languages is needed.

The remaining articles describe the capabilities and limitations of several existing or proposed systems. They discuss the types of information services provided, the way in which the files are set up, and the types of mechanical devices used. Equipment mentioned includes Minicards, Magnacards, the WRU Searching Selector, the IBM 7090, and the new GE-225 computer system with special features for performing searches.

The book is recommended to those who are currently working in some area touching upon information retrieval and to any others with a special interest in this fast-growing field.

Applied Mathematics Laboratory

Thomas S. Walton

David Taylor Model Basin

Washington 7, D. C. 